\title{
Körper/Leib als Schwelle der Übertragung im deutsch-französischen Verhältnis
}

Der Akzent und andere expressive Masken

Le corps (propre) comme seuil de transmission dans les relations franco-

allemandes : l'accent et d'autres masques expressifs

The Body as a Threshold of Transmissions in the German-French Relationship:

The Accent and other Expressive Masks

\section{Thomas Keller}

\section{(2)enEdition}

\section{Journals}

Édition électronique

URL : https://journals.openedition.org/ceg/9882

DOI : $10.4000 /$ ceg. 9882

ISSN : 2605-8359

\section{Éditeur}

Presses Universitaires de Provence

\section{Édition imprimée}

Date de publication : 20 janvier 2020

Pagination : 141-159

ISBN : 979-10-320-0253-7

ISSN : 0751-4239

Référence électronique

Thomas Keller, „Körper/Leib als Schwelle der Übertragung im deutsch-französischen Verhältnis“, Cahiers d'Études Germaniques [Online], 78| 2020, Online erschienen am: 28 Oktober 2021, abgerufen am 01 Februar 2022. URL: http://journals.openedition.org/ceg/9882 ; DOI: https://doi.org/10.4000/ ceg.9882 


\title{
Körper/Leib als Schwelle der Übertragung im deutsch-französischen Verhältnis
}

\author{
Der Akzent und andere expressive Masken
}

Thomas KELLER

Aix-Marseille Université, ÉCHANGES (EA 4236), Aix-en-Provence

Den Körper transkulturell, konkret: als Grenzbild und -objekt und -subjekt im deutsch-französischen Verhältnis einzusetzen ist mit Fallen besetzt. Allzuoft gerinnen Körper zu Allegorien von Nationen bzw. Kulturen (schwerfällig versus leicht u. Ä.). Auch kann die jeweils unterschiedliche Konjunktur des Körpers in Ländern, so die stärkere Verbreitung von Körperarbeit, Therapie, Tanztheater etc. bei Deutschen, zu essentialistischen Deutungen (etwa angebliches Fortwirken einer nicht-rationalen romantischen Tradition in Deutschland) missbraucht werden.

Das offenere Bild der „Schwellen“, schon von Benjamin vorgezogen, erlaubt es, lebendige und verdinglichte Physis einer transkulturellen Perspektive zu unterlegen. Der Körper/Leib ist ein Medium, das Franzosen und Deutschen für Übertragungen dient. Es wird zum berührbaren, berührenden und resonierenden Objekt und Subjekt. Indes finden auch auf dieser Schwelle immer wieder Grenzerfahrungen statt, etwa durch einen Akzent, der mir nicht nur leiblich eine Grenze der Integration in eine andere Sprache aufzeigt, sondern auch im plessnerschen Sinne ein Ausdruck eines grenzrealisierenden Lebewesens ist, das ein Leib ist und den Körper fernstellen kann - ich höre mich mit Akzent sprechen. Diese Grenze verläuft allerdings nicht zwischen Kulturen und zwischen Menschen, sondern in Menschen und Leibern.

Auch mein hier vortragender Körper/Leib ist ein solches Schwellen- bzw. Grenzmedium. Er ist lebendiger Leib, insofern er meine Stimme erklingen lässt, und er ist Körper, den ich habe, sofern ich meine Stimme selbst als getrennte, fremde höre. Ich setze voraus, dass auch die anwesenden Frankophonen Deutsch verstehen, die Sprache, in der ich vortrage. Dès que je passe au français, mon message devient accessible pour ceux dans l'auditoire qui ne comprennent pas l'allemand. Cependant, je ne prononce pas le français exactement comme un francophone. Je parle avec un accent. Cet accent, je l'entends moi-même. Je sais également que mon public le remarque et ressent ainsi la différence avec la prononciation d'un francophone possédant le français comme langue maternelle. L'oralité révèle un reste d'étrangeté. La transmission traîne un reste, une trace 
étrangère et étrange, qui transpose des propriétés de la langue allemande à la langue française. Mon parler porte la marque d'une légère différence, d'un écart, d'un désordre vocal.

Der Übertragungsprozess hat so etwas hinzugefügt, so etwas wie eine Unebenheit, einen stimmlichen Schorf. Die Frage nach der Besonderheit körperlich/leiblicher Erfahrung bei nicht-begrifflichen Übertragungen im deutsch-französischen Kontaktraum zieht jene nach der Beschaffenheit des Mediums nach sich: Es ist nicht bloßes Kommunikationsmittel wie das Telefon oder die E-mail, sondern bringt in einer besonderen, eben transkulturellen Weise, den Menschen als störanfälliges leibliches Wesen, als Mängelwesen ins Spiel. Im Akzent tritt diese irritierende Spur lautlich in die Wahrnehmung.

Ich versuche nun, zunächst eine kleine Theorie des Akzents zu formulieren. Weiter treibt mich die Frage um, was neben dem Akzent - vermittelt über andere Sinne als den Hörsinn - im Kontakt zwischen Kulturen körperlich beim Übertragen eingreift. Ich habe mich gefragt, ob es, analog zur abweichenden hörbaren Abweichung, also zur akustischen Maske, auch sehbare, riechbare gibt, so dass eine spezifisch transkulturelle expressive Maske entsteht. Dann möchte ich die Künste daraufhin abklopfen, welche Stellung sie diesen scheppernden sinnlichen Spuren einräumen.

\section{Kleine Theorie des Akzents: indizienhaft, defizient/supple- mentär, diakritisch, metonymisch, disjunktiv-integrativ, exzentrisch, pathisch, appelativ, affektiv unscharf}

Das auffälligste körperliche Anzeichen für eine transkulturelle Übertragung dürfte der Akzent sein. Besonders treffend und witzig erfasst die japanische auf Deutsch und Japanisch schreibende Autorin Yoko Tawada in ihrer Essaysammlung Akzentfrei den Akzent. Der Akzent ist „das Gesicht der gesprochenen Sprache“ ${ }^{1}$. Er ist ein „Migrationsvordergrund“2. Er „sickert in die Zweitsprache ein und erzeugt eine atonale Musik“3, so die musikologische Begrifflichkeit der japanischen Autorin, deren Bild vom „Gesicht der Sprache“ auch an die akustische Maske nach Canetti erinnert. Der Akzent ist zugleich eine Aufforderung zur Kreativität, da er „unerwartet zwei Wörter zusammenbringt, die normalerweise nicht ähnlich klingen“" . Tawada nimmt auch die rein klangliche Dimension des Akzents wahr. Sie erinnert auch, dass jemand, der akzentfrei Deutsch spricht, aber nicht aus Deutschland kommt, viele Schwierigkeiten im

1. Yoko Tawada, Akzentfrei. Literarische Essays, Tübingen, Konkursbuchverlag, 2016, S. 22.

2. Ibid., S. 27.

3. Die japanische Schriftstellerin Yoko Tawada im Gespräch mit Marietta Schwarz, Deutschlandfunk, 29.04.2018. Wolfgang Eßbach hat mich auf diese Sendung hingewiesen. Vielen Dank.

4. Tawada, Akzentfrei, S. 23. 
Alltag haben wird, da die Gesprächspartner fälschlich implizites Wissen (Begriffe wie „Einschreiben“"5) voraussetzen.

Hier sei eine kleine Theorie vorgeschlagen. Die Stimme gibt, neben dem klassischen Fahndungsbild, ein Musterbeispiel für das Indizienparadigma (Carlo Ginzburg) ab. Sprecher sind stimmlich in Geschlecht und Alter, oft auch dialektal und soziolektal markiert. Sie sind über Abweichungen erkennbar, so dass Oralität verräterisch wird. Bei der Suche eines Verbrechers wird mitunter eine Stimme über Radio und Fernsehen ausgestrahlt in der Hoffnung, dass ein Hörer die dazugehörige Person identifiziert. Inzwischen wird das kriminologische Verfahren der Stimmanalyse auch bei der Bestimmung von Herkunft und Alter von Flüchtlingen benutzt. Auch der Akzent in der Aussprache einer Zweitsprache macht Aussagen zur Herkunft (Region oder Ausland) möglich. Er eröffnet einen indirekten Zugang via Merkmale der Erstsprache. Dadurch entsteht eine besondere akustische Maske in Hinblick auf deutsch-französische Übertragungen, sie hinterlassen eine charakteristische Spur des jeweils anderen.

Im Sprachwechsel gewinnt das abweichende transkulturelle Anzeichen häufıg eine gewisse Stabilität und Regelhaftigkeit - in einem besonderen Akzent. Anhand von bestimmten Akzenten werden Sprecher - oft zu ihrem Leidwesen ,erkannt', ,durchhört', nämlich auf ihre Herkunft reduziert und einem Land zugeordnet, wobei sich die einzelnen Akzente unterschiedlicher Beliebtheit erfreuen. Der Akzent von Deutschen gehört sicherlich nicht zu den beliebtesten, der von Franzosen dürfte deutlich häufiger freundlicher aufgenommen werden. Seine Integration oder der Grad seiner Integration ist ungewiss. Wenn freilich ganze Gruppen von ihrer Institution gestützt mit verschiedenen Akzenten sprechen, können akzeptierte neue konventionale Varianten mit verschiedenen Akzenten entstehen, wie das Euro-Englisch in Brüssel.

Der Akzent wird zuallerst als defizient wahrgenommen. Ihm fehlt etwas, ein etwas, das die richtige Aussprache verhindert. Dieses etwas ist zugleich ein Zusatz. Der Akzent ist eine lautliche Variante des diakritischen Zeichens, mit dem er Kennzeichen teilt. Der Accent circonflexe speichert das verdrängte $S$ (château/chasteau). Er „reduziert“ und verbindet sich „mit einem anderen Buchstaben, einer anderen Instanz" ${ }^{6}$. Es verweist auf etwas, ohne es direkt zu benennen. ${ }^{7}$ Diakritische Zeichen wie Asteriske fügen in das Schriftbild einen Fremdheitswert ein. Das lesende Auge muss zur Dechiffrierung ein Vorwissen aktivieren. Diese Zeichen haben allerdings nicht - anders als der Akzent - einen Lautwert. Aber - wie das diakritische Zeichen - bewahrt auch der Akzent etwas von der abwesenden Erstsprache auf. Er nimmt nicht nur etwas weg, sondern fügt auch lautlich etwas hinzu, insofern er überträgt.

5. Ibid., S. 25.

6. Frank Witzel, Die Erfindung der Roten Armee Fraktion durch einen manisch-depressiven Teenager im Jahre 1969, Berlin, Matthes \& Seitz, 2016, S. 714.

7. Schachtheoretiker verweisen für diesen Vorgang auch auf die Bewegung des Pferds beim Schachspiel. Es schlägt die gegnerische Figur nicht in direkter Linie, es vollführt vielmehr eine Vorwärts- und Seitenbewegung und gelangt so als einzige Figur in einem einzigen Zug in eine Position, die den anderen Figuren nur über eine gerade Linie erreichbar ist. 
Der Akzent bezeugt meine Integration in eine zweite Sprache. Er stellt zugleich ein lautliches Signal dar, das die Erstsprache vertritt, die abwesend ist. Es handelt sich nicht um eine metaphorische Beziehung - der zweite Term setzt sich nicht an die Stelle des ersten -, sondern um eine metonymische Beziehung, die verschiebt. Die Benachbarung macht, dass die Besonderheiten des Erstkontextes, das ist hier die Aussprache in der Erstsprache, in einem phonologischen Transfer in die zweite gesprochene (Fremd)Sprache hinübergetragen ist. Der Akzent stellt so ein gespaltenes, ein disjunktives Anzeichen dar: ein Lautteil des Worts bewahrt Kennzeichen der Erstsprache auf, während der andere Lautteil auf die Zweitsprache verweist. Die Stimme zeigt die fremde Herkunft unabhängig vom semantischen Gehalt. Der Akzent selbst verweist auf kein besonderes Signifikat. Diese Eigenschaft der transkulturellen Übertragung stellt eine besondere abweichende Beziehung zwischen dem Ausdruck und dem Ausgedrückten her. Die Stimme enthüllt eine physiognomische Seite der Übertragung. Diese Spur der Übertragung bildet ein irritierendes Drittes, das den sprechenden Körpern anhaftet. Es bildet ein transkulturelles Feld, in dem das Verstörende einen sinnlichen und materialen Widerstand gegen die glatte Kommunikation aufbietet. Er verbindet und trennt zugleich Menschen und Kulturen.

Um die eigenartige Position der unterschiedlich störenden Stimme zu beschreiben, kann man den Begriff des disjunktiven Anzeichens auch mit dem der „disjunktiven Integration“ von Pierre Klossowski ${ }^{8}$, oder der „disjunktiven Synthese“ von Gilles Deleuze/Félix Guattari ${ }^{9}$ ergänzen, mit dem sie das ausschließende Postulat der Widerspruchsfreiheit umgehen. Hier sei er verwendet, um das nicht-identitäre Element zu entschlüsseln, das in und zwischen mehreren Kulturen gleitet und immer beides bleibt, das eine und das andere ist und es nicht ist. Es ist jene Möglichkeit der Widersprüchlichkeit, die jene Eigenschaft der disjunktiven Integration bewahrt. Ein deutscher Akzent im Französischen verortet weder eindeutig im Französischen noch im Deutschen und verbindet doch beide.

Jene Irritation steht quer zur Annahme, die Stimme sei allein ein Medium der Wortsprache $^{10}$. Die semiotische Seite dieses Vorgangs ist zugleich eine leibliche.

8. Den Begriff „disjunktive Integration“ entwickelt Klossowski, um das Verhältnis von Nietzsche und Dionysos offen zu lassen. Er bezeichnet eine „vertikale“ Mensch/Gott-Konstellation: „Dans Le Baphomet, Klossowski opposait à Dieu comme maître des exclusions et limitations dans la réalité qui en dérive, un antéchrist, prince des modifications, déterminant au contraire le passage d'un sujet par tous les prédicats possibles. Je suis Dieu je ne suis pas Dieu, je suis Dieu je suis Homme [...] [I]l s'agit d'une disjonction inclusive qui opère elle-même la synthèse en dérivant d'un terme à l'autre et suivant la distance. Il n'y a rien d'originaire“, Gilles Deleuze, Félix Guattari, L'Anti-CEdipe. Capitalisme et schizophrénie, Paris, Minuit, 1972, S. 92.

9. Deleuze/Guattari bezeichnen damit eine horizontale Mensch-Mensch-Konstellation, diejenige des Schizos: „chacun passe dans le corps de l'autre sans organes“, Deleuze, Guattari, L'AntiEdipe, S. 29. Der Schizo verwirklicht die „disjunktiven Synthesen“ des „sowohl als auch“ („les synthèses disjonctives“ du „soit... soit“), ibid., S. 18.

10. Vgl. Sybille Krämer, „Die „Rehabilitation“ der Stimme. Über die Stimme jenseits der Oralität“, in Sybille Krämer, Doris Kolesch (Hrsg.), Die Stimme, Annäherung an ein Phänomen, Frankfurt a. M., Suhrkamp, 2006, S. 269-295. 
Das Sprechen mit Akzent ist eine Handlung, die eine Schnittstelle zwischen Sprache und Körper ausbildet: Eigenarten der Erstsprache, nämlich motorische eingeübte Fähigkeiten von Lippe, Zunge, Kiefer, Stimmritze, Stimmband, Kehlkopf, Thorax, Zwerchfell kommen in der Zweitsprache zum Einsatz und verändern, verzerren sie. Übertragen auf die Zweitsprache bedarf die Aussprache einer besonderen Anstrengung und droht, der Kontrolle zu entgleiten. Insofern ,erleidet ' der mit Abweichungen Sprechende seinen Akzent. Der Akzent verstärkt die pathische Eigenschaft der Stimme - beim Sprecher und beim Hörer.

Im Kulturkontakt treten Sprecher und Zuhörer in ein asymmetrisches Spiel ein. Auch die Zuhörer nehmen den Akzent als eine stimmliche Unebenheit wahr, sie ,erleiden“ ihn. Sie ist eine mehr oder weniger vernehmbare Abweichung, eine ,Unordnung“, die aus Eigenschaften der Erstsprache herrührt. Es handelt sich um eine physisch-psychische Erfahrung während der Übertragung, die - anders als bloße Kommunikationsmittel wie das Telefon oder die E-mail - eine riskante Form von Medialität verkörpert, deren Abweichung etwas Ungefähres eigen ist. Sie ist eine Spur, die ihre Wirkung entfaltet, bevor ein Verstehen und Austausch von Argumenten einsetzt.

Der Akzent potenziert die Erfahrung von exzentrischer Positionalität. Wie jeder Mensch erfährt sich der mit Akzent Sprechende als Körper, den er hat, und als Leib, der er ist. Aber nur er weiß um seinen Akzent und er hört ihn mit; er weiß um die Reaktion des Hörers, für den der Akzent eine so überraschende wie irritierende Abweichung ist. Insofern wir uns sprechen hören, machen wir eine Fremderfahrung. Ich berühre mich, ich höre mich, und ich werde berührt in einer Bewegung der Entfremdung. Die Selbsttranskription - ich messe meiner gesprochenen und gehörten Stimme eine Botschaft zu, ich springe zwischen verschiedenen Ebenen, zwischen Körper und Gedanken, in einem gleichsam metaleptischen Verfahren ${ }^{11}$ - findet im Akzent eine Grenze. Der Akzent überträgt nicht semantisch Informationen, sondern signalisiert Fremdheit. Insofern ich mich nicht nur sprechen höre, sondern auch mit einem Akzent sprechen höre, nimmt die Entzweiung mit mir selbst eine zusätzliche Dimension an. Besonders ,peinlich“ ist die von einem Tonträger aufgenommene eigene mit Akzent sprechende Stimme. Nicht nur die Gedanken werden als vom Körper losgelöst wahrgenommen, die abweichende Stimme bleibt unintegriert in die lautliche Gemeinschaft. Sie widersteht der Reziprozität, sofern mein Gesprächspartner akzentfrei spricht. Auf der Ausdrucksseite entsteht ein besonderer Drittkörper der letztlich nicht-integrierbaren Verzerrung. Angesichts dieser Asymmetrie ist das Sprechen mit Akzent ein Zeugnis des Fremden. Die Rede vom Mängelwesen Mensch bekommt hier eine eigene leibhafte Dimension.

Der Akzent kann nicht als Rolle aufgefasst werden, die bewusst übernommen ist. Mittels Akzent zieht mein lebendiger Leib, ob ich will oder nicht, eine mir selbst hörbare Grenze ein: Ich kann in der körperlichen Hülle des Zweitkontextes die Abweichung, das Anzeichen meiner Herkunft, nicht einfach abstreifen. Dies

11. Vgl. Erika Linz, „Die Reflexivität der Stimmme“, in Cornelia Epping-Jäger, Erika Linz (Hrsg.), Medien/Stimmen, Köln, Dumont, 2003, S. 50-64, hier S. 54. 
gilt für die dialektale bzw. soziolektale Prägung wie auch für den Akzent, der die Muttersprache verrät. So kann ein westfälischer Zungenschlag doppelt, zunächst in der Aussprache der deutschen Hochsprache, dann im Akzent im Französischen, durchschlagen.

Hier liegt meine Grenze und auch meine Invalidität, meine Verletzlichkeit, meine ,Natürlichkeit: Zwar hindert mich nichts, mit Akzent zu lügen und zu täuschen. Aber der Laut selbst ist meiner Kontrolle entzogen, er ist unwillkürlich und macht sich selbständig. Er bewegt sich in der Nähe von Peinlichkeit und Kontrollverlust. Er ist weder unmittelbare Lebensäußerung noch ganz rational gesteuert, weder Werk noch Bewusstsein, wohl aber eine Ausdrucksleistung, die zugleich Eigenschaften von Künstlichkeit hat. Es ist wohl kaum - wie bei Lachen und Weinen - möglich, mittels Akzent Luft abzulassen, sich emotional zu befreien. In der Wahrnehmung des Sprechers wie des Zuhörers gibt die andere Aussprache, der andere Stil dem mehr oder weniger spontanen expressiven Fluss etwas Gebrochenes ein. Der Kulturwechsler ist mit dieser Verkörperung ,geschlagen: Er kann sich angesichts dieser Ungleichheit wünschen, dass die Abweichungen zwar wahrgenommen, nicht aber Anlass zu Urteilen über ihn gibt. Seine Stimme wird dann ein Anruf um Wohlwollen. Seine Verkörperung ist das Medium, mit dem sich die Probe auf Aufnahme ereignet.

Der Akzent gehört den prekären sprachleiblichen Medien an, die transkulturelle Übertragungen affektiv unscharf machen - auf Seiten von Sprecher und Hörer. Der Sprecher kann den Akzent sehr selbstbewusst vertreten oder eher schambesetzt erleiden ${ }^{12}$. Der Hörer kann einen Akzent neutral, mit Wohlwollen aufnehmen oder mit Ablehnung bis zur Feindseligkeit reagieren. Der Akzent birgt ein Potential von Verlust von Selbstvertrauen auf Seiten des mit Akzent Sprechenden wie von Misstrauen auf Seiten des Hörers.

Spricht jemand eine Zweitsprache ohne Akzent, kann dies einer mehrkulturellen Familienkonstellation entsprungen, eine perfekte assimilatorische Anpassungsleistung wie auch eine Verstellung sein, die dazu dient, feindliche Absichten, etwa die Beauftragung durch eine fremde Macht, also Spionage, zu camouflieren (Mimikry). Für den Hörer kann die Abwesenheit des Akzents bei gleichzeitigem Wissen um fremde Herkunft Indiz für Integration wie auch eine beunruhigende Chamäleonhaftigkeit darstellen. Der Akzent ist nicht nur affektiv unscharf, er unterliegt auch dem Momentum des Kippens. Die transkulturelle physiologische Herübernahme ist eine besondere Manifestation von kreatürlichen unwillkürlichen Regungen, die in unterschiedlichem Ausmaß auffällig bzw. unauffällig, ähnlich bzw. unähnlich sind.

Der Akzent macht die übermittelte Information nicht nur unklarer, uneindeutiger, ungefährer; er löst auch von allem Sinn ab und wird reiner Laut, ein sinnlich-materialer Überschuss. Man kann diese transkulturelle Expressivität auch als unverwechselbare hörbare „akustische Maske“

12. Jacques Derrida schildert in Le Monolinguisme de l'autre (Paris, Galilée, 1996), wie ihm die meridionale Aussprache des Französischen wie der Pieds-Noirs-Akzent peinlich ist und er sie konsequent und gezwungen vermeidet. 
bezeichnen (ohne die negative Konnotation bei Canetti zu übernehmen), die irritiert wie auch autonomisiert. Der Akzent ist die persona der Migration, nicht die Maske der Verstellung, sondern die typische Ausdrucksform von durchtönender grenzüberschreitender Mobilität. Unverwechselbare Akzente sind das Unübersetzbare, das Unvertretbare. Die Formel der Philosophischen Anthropologie, in seiner gespielten Rolle sei der Mensch ersetzbar, nicht aber in nicht-gespielten unwillkürlichen leiblichen Regungen wie Lachen und Weinen gewinnt in Hinblick auf leiblich vermittelte Transkulturalität ein weiteres Feld. Im transkulturellen Kontext verrät der Akzent keine dialektale oder soziolektale Besonderheit, sondern verwirklicht eine lautliche Migration, die Grenzen zwischen Wörtern verschiebt. Die veränderte Aussprache markiert nicht nur eine Abweichung, sie spielt auch neue Nachbarschaften von Wörtern $\mathrm{zu}^{13}$. Hier entsteht ein kreatives Feld des Sprachartisten.

\section{An- und Abwesenheit des Akzents in der Literatur}

Das Indizienparadigma ist in verschiedenen Medien wirksam, in Stimme, in Bild (das berühmte Fahndungsfoto) und auch im Stil. In der Literatur hingegen dürfte es nur sehr wenige Beispiele für die Präsenz des Akzents geben. Dafür ist eine Transkription nötig, die in der Schrift, in einer abweichenden Orthographie, den Akzent nachäfft und den Sprecher schnell der Lächerlichkeit aussetzt, also ganz den diskriminierenden Effekt ausschlachtet. Eine Scheu, dem anderen einen Gesichtsverlust zuzufügen, greift ein. Es kommen freilich auch vereinzelte beiläufıge literarische Einsätze des Akzents vor, die eine anziehende Wirkung haben. ${ }^{14}$

Auch die schriftliche Unauffälligkeit, die stilistische Brillanz kann den Dienst zugunsten einer fremden Macht verschleiern. In der Literatur ist das Erstaunen über den abwesenden Akzent leichter aufzufinden als Demonstrationen seiner Anwesenheit. Der abwesende Akzent kann Verschiedenes andeuten. So wechselt Corinne in Mme de Staëls gleichnamigem Roman von 1806 in der Begegnung mit Lord Oswald plötzlich von ihrem muttersprachlichen Italienisch in ein akzentfreies Englisch. Die Irritation geschieht, als Corinne ihre Krone verliert,

13. Yoko Tawada gibt die Annäherung von „Zelle“ und „Seele“ an, wenn diese Wörter von einem japanischen Muttersprachler ausgesprochen werden. Tawada, Akzentfrei, S. 23.

14. Im Zauberberg imitiert die Schreibweise den russischen Akzent von Madame Chauchat. Sie sagt in bezug auf den Humanisten Settembrini: „er war nicht menschlich gesinnt. Die Stimme sprach das Wort „mähnschlich“ aus, mit einer gewissen trägen und schwärmerischen Drehung.“ Thomas Mann, Der Zauberberg, Frankfurt a. M., Fischer, 2002, Große kommentierte Frankfurter Ausgabe, Band 5.1., S. 843. „Mähnschlich“ umfasst alle Dimensionen des Menschen, auch die Leidenschaften. Der Akzent rückt auch vom rein Vernünftigen, von deutscher Eingeschränktheit, weg. Er korrespondiert der trägen Körpersprache. Zugleich bewirkt er, im Bunde mit dem vielsagenden Eigennamen Chauchat und der Geste des Überreichens des crayon, eine stimmliche Erotisierung. „Mähnschlich“ ist allerdings das einzige lautmalerische Wort im Text. Madame Chauchat spricht ansonsten deutsch und französisch durcheinander. An dieses Beispiel hat Hélène Barrière mich erinnert. Ähnlich wirkt der englische Akzent der Schauspielerin Jane Birkin im Französischen. 
Oswald sie aufhebt und ihr reicht und sie ihm in einem akzentfreien „nationalen“, „reinen“, „insularen“ Englisch dankt ${ }^{15}$. Nicht der Akzent im Englischen, sondern seine Abwesenheit macht Corinne in ihrer italienischen Umgebung fremd. Die Zweisprachigkeit ohne Akzent macht sie geheimnisvoll und nichtintegrierbar. Es taucht allerdings kein Englisch wörtlich im französischen Text auf. Die akzentfreie Aussprache macht die Protagonistin unheimisch. Sie wird an der Position zwischen Kulturen zerbrechen. De Staël inszeniert in Corinnes pluralen Sprachleib einen stimmlichen Kulturkonflikt; sie betrauert zugleich die Unmöglichkeit, zwei Kulturen zu vereinbaren.

Ganz anders als de Staël arbeitet Jean Giraudoux in Siegfried et le Limousin mit der Anwesenheit bzw. Abwesenheit von sprachlichen Kennzeichen. ${ }^{16}$ Wir befinden uns im Zeitalter der konkurrierenden Nationalstaaten. Der Leib des gedächtnislosen Jacques Forestier wird auf dem Schlachtfeld von Deutschen aufgefunden und gesundgepflegt. Aus ihm wird Siegfried von Kleist. Die Handlung besteht nun darin, aus dem verschobenen Leib wieder einen Franzosen zu machen. Die Hauptfigur Forestier/Kleist spricht Deutsch und Französisch ohne Akzent. So scheint die Sprache es nicht zu erlauben, ihn als Franzosen oder Deutschen zu identifizieren. Die Person bewahrt indes jenseits der Übertragung unveränderte Züge. Es sind bezeichnenderweise zunächst nicht körperliche Merkmale, sondern stilistische sprachliche Kennzeichen, die es ermöglichen, ihn zu identifizieren, nämlich der Gebrauch der Litotes in von Forestier/Siegfried auf Deutsch geschriebenen Texten. Dieses angeblich französische Stilmerkmal kann über weite Entfernungen auffallen, so dem Leser der Frankfurter Zeitung in Paris, der einen SvK gezeichneten Artikel liest. Es bezeugt die mögliche schriftliche Übertragung vom französischen auf ein deutsches Sprachcorpus.

Ein weiteres Indiz fällt dann in direkter Begegnung und Beobachtung zu. Siegfried von Kleist lernt problemlos Französisch, spricht schnell akzentfreies Französisch und beherrscht sofort perfekt den subjonctif imparfait.

Die Kennzeichen des Jacques Forestier sind nicht kontingent. Die laufende Handlung von Siegfried zieht die Grenze wieder ein. Der Prozess ist zweifach disjunktiv und integrativ, in einer kreisförmigen Bewegung wird aus Jacques Forestier Siegfried von Kleist, aus Siegfried von Kleist wieder Jacques Forestier. Allerdings ist die Bewegung nicht beliebig weiter verlängerbar. Der nackte gedächtnislose Körper bewahrt Kennzeichen von Jacques Forestier auf, sie sind ins Deutsche verschoben. Die in der deutschen Lebensphase erworbenen Kennzeichen hingegen können nicht dieselbe identitäre Qualität beanspruchen, insofern auch nicht verschoben werden. Der im Indizienprozess identifizierte Mann wird als Franzose wohl kaum an Kennzeichen aus deutschen Kontexten

15. Germaine de Staël-Holstein, Corinne ou l'Italie (1807), Paris, Gallimard (folio), 2003, S. 68. „Corinne remercia lord Nelvil, en anglais, avec ce pur accent national, ce pur accent insulaire qui presque jamais ne peut être imité sur le continent.“ [„Corinna dankte Lord Nelvil auf Englisch mit einer ganz reinen Aussprache und dem eigentümlichen Akzent, der fast nie von Ausländern nachgeahmt werden kann." Germaine de Staël-Holstein, Corinna oder Italien, München, Winkler, 1979, S. 42, übersetzt von Dorothea Schlegel].

16. Jean Giraudoux, Siegfried et le Limousin, Paris, Grasset, 1922 (Prosafassung). 
festhalten. Die Merkmale, die auf Deutsches verweisen, sind revidierbar. Kleists Körpergeste, das Hacken zusammenschlagen beim Grüßen, das Übertreiben in seinen deutschen Texten, wird der wiedergefundene Jacques Forestier nicht fortsetzen.

Die Litotes, also die Untertreibung, das Bewusstsein für das Maß, schlägt sich jenseits der Sprache auch körperlich und im Bild vom Körper - physiognomisch und physiologisch - nieder: keine Grimasse, kein starkes Gestikulieren, kein Schreien. Die unmittelbar physische Dimension tritt im Siegfried nach den sprachlichen und künstlerischen expressiven Indizien ein.

Zusätzlich $\mathrm{zu}$ der stilistischen und stimmhaften Indizienhaftigkeit der Hauptfigur Forestier/Kleist im Siegfried ist der Ich-Erzähler Gegenstand einer bildlichen Übertragung. Er taucht in der Form einer ontologischen Metalepse in einem Bild von Poussin auf, das von München nach Paris gelangt. Auch hier ist damit eine Aufforderung an Identifikation verbunden. Der verblüffte Betrachter seines Porträts soll sich an die identifikatorische Aufdeckung des Geheimnisses, also aus Siegfried wieder Jacques machen.

Physiognomische Anzeichen - etwa Narben - verraten. Das Indizienparadigma ist zugleich ein Odysseus-Skript. Es setzt sich stilanalytisch, bildlich und olfaktorisch durch. Neben dem Hören und Sehen kann die Körpergrenze auch durch Riechen in identifikatorischer Absicht bestimmt werden. Die mythologische Szene von Odysseus' Rückkehr nach Ithaka aufgreifend erkennt das Tier, hier der Hund Black, seinen Herrn Forestier am Geruch. Dies setzt die leibliche Begegnung von Mensch und Tier voraus. Der Hund muss nach München geschafft werden. Die unmittelbares Erkennen versprechenden Sinne, das Wiedererkennen Forestiers mit dem Auge sowie seine olfaktorische Identifikation, werden in praesentia eingesetzt.

Der Indizienprozess ist identitär. Er macht die Integration in ein deutsches Milieu rückgängig. Letztlich basiert die Handlung auf fixer Ähnlichkeit der Personen mit einer Nation und Kultur. Das kulturelle Skript ist griechisch-christlich: Odysseus ist an der Narbe und am Geruch nach seiner heimlichen Rückkehr nach Ithaka erkennbar; das Skript von Christus und Thomas dem Ungläubigen ${ }^{17}$ ist variiert: Auch die Hauptfigur Siegfried ist ein Wiederauferstandener, der stimmlich, stilistisch und grammatikalisch sowie sinnlich-leiblich berührt und damit vereindeutigend bezeugt wird.

Die „Aufklärung“ von Identität geht nur in eine Richtung, in Richtung Frankreich. Der Text bleibt identitär. Der Körper als Grenze bleibt ein Grenzkörper. Das Resultat ist paradox: Nicht der Akzent zieht eine Grenze zwischen Kulturen ein, sondern seine Abwesenheit.

Die Ambivalenz von Auffälligkeit und Unauffälligkeit, von Mangelhaftigkeit und Perfektion, bestimmt nicht nur die Oralität. Die Übersetzung scheint den Körper, der Abweichungen zum Ausdruck bringt, zum Verschwinden zu bringen.

17. Siehe hierzu Jacques Derrida, Le Toucher. Jean-Luc Nancy, Paris, Galilée, 2001. Derrida sperrt sich gegen den Kurzschluss von Ertasten der Wunden mit dem Herzgefühl, gegen eine vorgebliche Authentizität, in der Berühren, Fühlen und Seele zusammenfallen. 
Indes, wenn sie auch keinen Akzent kennt, so kann sie doch durch Stilfehler, durch einen Lapsus verräterisch sein. Auch in der Theateraufführung und im Vortrag etwa eines Gedichts kann die abweichende Aussprache gezielt eingesetzt werden. Verkörperung findet statt.

Der Akzent stellt nur einen Aspekt der expressiven Maske dar, die im transkulturellen Kontakt Merkmale des Erstkontextes herüberträgt und so einen sinnlich-materialen Überschuss erzeugt. Ist es beim Akzent der Hörsinn, der fremdstellt, so kann ver- und befremdende exzentrische Positionalität auch über andere Sinne entstehen; so wenn ich mich selbst sehe und sehe, wie andere mich sehen; so wie ich mit Fehlern und mit stilistischen Abweichungen schreibe und dabei lesbare Merkmale meiner Muttersprache mitbringe. Die Spur kann lautlich, bildlich und auch sächlich (ein nicht-lebendiger Gegenstand) sein. Im Stil zeigt sich eine sichtbare wie auch lesbare Marke. Neben der phonologischen und stilistischen Übertragung im Akzent und im Stilbruch ereignen sich auch bildnerische Herübernahmen. Auch Körpergesten und Kleidung speichern wahrnehmbare Codes des Erstkontextes. Im herübergenommenen Bildelement sieht der Betrachter zweierlei, Herkunft und Neukontextualisierung.

Die mal einschließende Wirkung, mal ausschließende Wirkung von Übertragungen betrifft die gesamte expressive Maske. Im transkulturellen Kontakt wirken disjunktive Anzeichen, ob lautlich, schriftlich oder bildnerisch (plastisch) als Masken. Sie machen Menschen zu personae. Stil und Bild bilden eine les- und sehbare Grenze, die undurchlässig oder durchlässig sein kann. Sie können lügen und sind doch ,natürliche“ Äußerungen, in denen sich der Ausdruck vom Inhalt ablöst und zum reinen Laut, Buchstaben oder Bild wird. Sie entziehen sich der vollständigen Vereinnahmung im Zweitkontext und zeichnen ein Unübersetzbares, ein Unübertragbares aus.

Der steilen Alternative von Auffälligkeit und Unauffälligkeit, von Abweichung und womöglich heimtückischer Ähnlichkeit, möchte ich schließlich eine andere Wahrnehmung entgegenstellen. Das, was als akustische oder stilistische Maske bezeichnet wird, die Laute und Buchstaben durchtönen und scheppern lässt, hat ein Äquivalent im „Mitriss“ bildnerischer Kennzeichen, die gleichgültig lassen, entzücken oder befremden.

\section{Der Mitriss des Bildaktes: der Stil der Friedensordnung}

Auch Bilder können dazu dienen, Menschen zu identifizieren. Das Fahndungsfoto oder das Phantombild machen dies deutlich. Solche Bilder haften für fassbare ,Identität: Sie zielen auf die Verhaftung des Gesuchten ab, sofern Abbild und Original übereinstimmen. Das ,fremdländische“ Aussehen löst dabei häufig antipathische Regungen bei Betrachtern, bzw. eine Verweigerung von Empathie aus. Eine Grenze zwischen Menschen und zwischen Gruppen bildet sich.

Indes, Bildakte können auch desidentifizieren und dadurch wandern. Bestandteile von Bildern wie Farbe und Form können intrinsensische Elemente von Bildakten sein, die zwischen Kulturen übertragen, einen „Mitriss der Form“ 
vollziehen ${ }^{18}$. Der Begriff „epigonal“ wertet jenen Vorgang allzu schnell ab. Das Porträt der Wilhelmine von Cotta, von Gottlieb Schick 1802 gemalt, greift die Position von Juliette Récamier auf, wie sie der schwäbische Maler kurze Zeit vorher in Paris auf den Bildern von David und Gérard beobachtet hat. Er schafft eine Ähnlichkeit und Unähnlichkeit zwischen der französischen und deutschen Grazie. Schick ersetzt den antikisierenden Hintergrund durch ein natürliches Dekor, das rote Tuch verdeckt nicht wie bei Gérard eine antikisierte Landschaft. Er platziert die deutsche Grazie in eine urwüchsige Umgebung, wie sie am Neckar mit seinen Baumbeständen bei Tübingen typisch ist ${ }^{19}$.

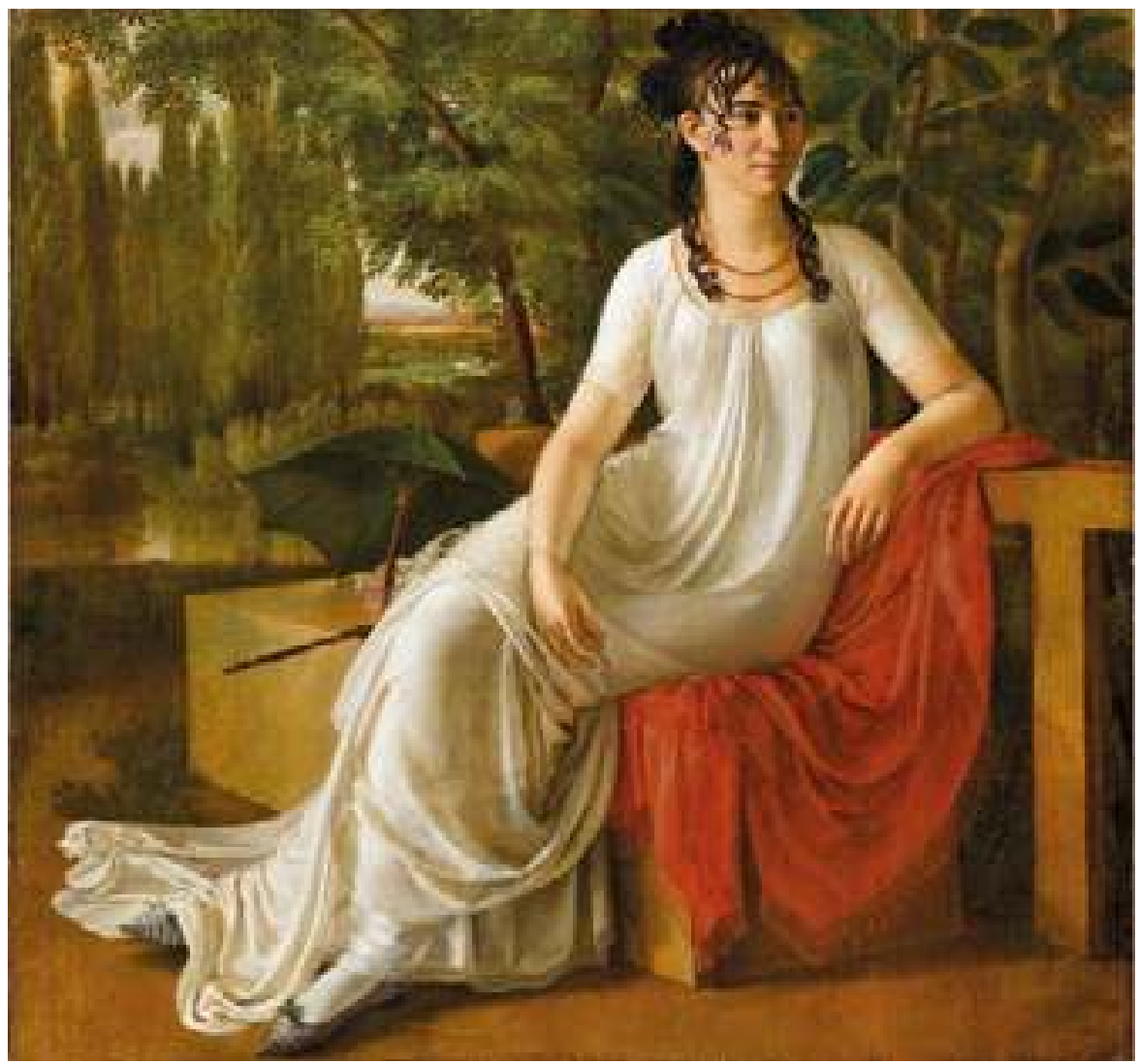

Fig. 1 : Gottlieb Schick, Wilhelmine Cotta, 1802; InvNr. GVL 87; Ölgemälde, 133,0 x 140,5 cm, Staatsgalerie Stuttgart, Foto @ Staatsgalerie Stuttgart

18. Horst Bredekamp, Der Bildakt, Berlin, Wagenbach, 2015, S. 277-299.

19. Dankenswerterweise genehmigt die Staatsgalerie Stuttgart die kostenfreie Abbildung des Porträts - während das Musée Carnavalet, in dem Gérards Porträt von Madame Récamier hängt, Gebühren fordert. 
Selbst das Rot nimmt einen ähnlichen Platz in den beiden Bildern ein. Die Übertragung verwirklicht ein Durchscheinen der Form. Der neoklassizistische Stil stellt eine Verbindung her. Beide Frauen folgen der Mode mit den Korkenzieherlocken, dem kurz unter der Brust zusammengerafften Gewand. Unterschiede bestehen in der größeren Laszivität von Madame Récamier, sie zeigt ihre nackten Füße vor, ihr Dekolleté ist etwas gewagter. Madame Cotta trägt karierte spitze Schuhe. Das später Récamière genannte geschwungene Möbelstück, auch für ein Schäferstündchen verwendbar, ersetzt Schick durch einen quadratischen Block. Aber hier einen Gegensatz von französischer Verführerin und deutscher Tugendwächterin zu sehen, wäre ganz verfehlt. „Mitgerissene“, also herübergetragene sprachlose bildnerische Elemente wie Form und Farbe vermeiden, umgehen jegliche physiologische und physiognomische Erfahrung von Fremdheit, wie sie etwa die Stimme verkörpern kann.

Man könnte annehmen, dass das Bild des Deutschen ,romantischer' ist, die Naturidylle scheint dies nahezulegen. Aber hier einen Gegensatz von Romantik und Klassik zu konstruieren, geht gänzlich an der Sache vorbei. Der mögliche Mitriss steht hier für einen transkulturellen Raum. Die Übertragung löst keine abwehrenden Gefühle aus. Die Verbindung der jeweiligen Verkörperungen im Bild bewirkt keine Grenzziehung, wenngleich sie nicht auf Identifızierungen des einen durch den anderen aus sind. Vielmehr bringt ein Spiel von Ähnlichkeit und Unähnlichkeit die beiden Bilder, die beiden Frauen, Württemberg und Paris in Kontakt. Das Porträt bezeugt formal, stilbewusst, ein Interesse für Paris, für die europäische Hauptstadt. Die Übertragbarkeit spiegelt einen deutschfranzösischen Raum unter Napoleon, der noch voller Hoffnung ist. Das Bild verzweigt sich zumindest für den informierten damaligen Betrachter kontextuell mit den Publikationsprojekten von Johann Friedrich von Cotta, dem Tübinger Verleger etwa der Französischen Miscellen, die wenig später für das deutsche Publikum aus Paris berichten. Dass Madame Récamier wenig später in Coppet Bündnispartnerin von Mme de Staël gegen Napoléon wird, ist hier nicht erkennbar, zumindest nicht relevant. Der mögliche Mitriss situiert Schicks Porträt in eine Zeit unmittelbar vor dem nationalen Erwachen von Deutschen. Noch markiert das Bild keine Grenze. Vielmehr vollzieht es eine stilistische Angleichung durch mobile Elemente.

Der Mitriss im Stil entspricht der napoleonischen Friedensordung, die einige Jahre dauert und dann vor allem durch Napoleon selbst zerstört wird. Der Betrachter kann das Bild nur nachträglich in feindseliger Absicht wahrnehmen und deuten, nämlich in denunziatorischer Weise einen Import fremdländischer Mode wittern. Und einige wenige Jahre später, in den anti-französischen „Befreiungskriegen“, geschieht Entsprechendes, etwa, wenn altdeutsche Tracht gefordert wird. Eine Körper-Grenze wird eingezogen.

Der stilistische Mitriss auf dem Bild von Schick ist weder störend noch pathisch noch defizient. Intrensisch bedeutet hier, dass das Vorbild der Mode sich ausbreitet, ohne selbst ein Feld des Widerstands auszubilden. 


\section{Die Patheinformel in transkulturellen Körperbildern: Krieg}

Meine Frage war, ob es „störende“ übertragende expressive Masken auch in der Form der bildenden Kunst gibt. Die bildnerische Maske stößt schnell an eine kulturelle Grenze, wenn etwa starke Emotionen sich in extremer angeblich übertreibender Mimik und Gestik, etwa in der Grimasse, verkörperlichen und Gefühle der Peinlichkeit hervorrufen. Und doch wandert zwischen den Avantgarden in Deutschland und Frankreich ein Bildreservoir des Erleidens, eine plastische Maske, wofür ich in Anlehnung an die „Pathosformel“ nach Aby Warburg den Begriff „Patheinformel“ verwenden möchte ${ }^{20}$. Eigenschaften des Sprechens mit Akzent wie Defızienz, Erleiden, affektive Unschärfe kennzeichnen auch gewisse transkulturelle Bildakte. Warburg hat für diesen Prozess des Wanderns von Bildelementen zwischen Bildern aus verschiedenen Epochen und Räumen einen Begriff geprägt. Er spricht von „Bilderfahrzeugen“ “21, die Interaktion zwischen verschiedenen Aufnahmekontexten verwirklichen. Beispiele für transkulturelle Bildakte lassen sich in den Avantgarden des 20. Jahrhunderts auffinden, etwa bei Max Ernst, André Masson und Victor Brauner.

Zwischen avantgardistischen deutschen und französischen Kontexten wandern expressive Masken, die stark irritieren. Auf den Bildern von Max Ernst tauchen die erhobenen Hände wie auch der leere oder in die Ferne schauende Blick von Fisch, Vogel, Mensch jenseits der kulturellen Grenze auf. Von der Chinesischen Nachtigall (1920) über Ubu imperator (1923) zu den furchtbaren Monster-Engelfiguren (etwa der Hausengel von 1937) der dreißiger Jahre bilden sie eine Reihe von Bildakten, die eine durchgehende Patheinformel bezeugen und dem Betrachter einen ihn verstörenden Anblick bieten. Sie durchschreiten mühelos die Grenze zwischen der dadaistischen Collage und der surrealistischen Traumszenerie.

Die chinesische Nachtigall ist ein Märchen von Hans Christian Andersen. Am chinesischen Kaiserhof kann eine künstliche Nachtigall nur scheinbar die lebendige ersetzen. Nur die natürliche Nachtigall spendet dem Kaiser von China Trost beim Sterben. Die Technisierung im Krieg hat ihre Spuren in Max Ernsts Foto-Montage hinterlassen. Das zugrundeliegende Foto stammt aus Georg Paul Neumann, Deutsches Kriegsflugwesen von 1917 über Flugwaffen. ${ }^{22}$ Es stellt eine englische Bombe dar. Der chinesische Fächer weist auf das Märchen zurück. Der konische Kopf in der Mitte ist Vogelkörper wie auch Flugkörper, also ein Projektil, das explodieren soll. Die Foto-Montage war für die Publikation Dadaglobe vorgesehen, mit der Tristan Tzara ein internationales Spektrum des Dadaismus vorstellen wollte. Dieses Buch ist nie verwirklicht worden.

20. Siehe Dieter Wuttke, Peter Schmidt, Aby Warburg und die Ikonologie, Wiesbaden, Harrassowitz, 1993.

21. Andreas Beyer, Horst Bredekamp, Uwe Fleckner, Gerhard Wolf (Hrsg.), Bilderfahrzeuge, Aby Warburgs Vermächtnis und die Zukunft der Ikonologie, Berlin, Wagenbach, 2018.

22. Georg Paul Neumann, Deutsches Kriegsflugwesen, Bielefeld, Velhagen und Klasing, o.J. [1917]. 
Entscheidend ist die Körpergeste, die erhobenen Hände, die Ohnmacht signalisieren oder vor einer Bedrohung oben schützen soll. Sie spiegelt die Traumatisierung infolge des Krieges. Diese Körpergeste wie auch der zwischen organisch und anorganisch, zwischen Vogel und Kriegswaffe schwankende Körper bzw. Leib gehen über die Grenze.

Max Ernst annulliert den versöhnlichen Schluss von Andersens Märchen keine natürliche Nachtigall ist zum trostspendenden Gesang in Not und Tod zur Stelle. Die dadaistische Nachtigall ist dauerhaft technisiert.

Was nun überträgt, geht über die Grenze? Es ist die Versehrtheit und die Ohnmacht, die Wut auslösen. Die erhobenen Hände tauchen in weiteren Werken auf. Im Jahre 1923 schafft Max Ernst seinen Ubu imperator. Ernst ist kurze Zeit vorher von Köln nach Paris übergesiedelt. Im Bild ist aus dem König Ubu (roi Ubu) von Jarry ein Imperator geworden. Die sehr bekannte runde Form des Père Ubu, an den lächerlichen Lehrer Hébert von Jarry erinnernd, ist erhalten und verändert zugleich. Die Stelzenbeine sind ersetzt durch einen Kreisel. Ubu ist im Wortsinne nach unten zugespitzt. Er droht jeden Moment umzufallen. Der Körper ist aus Behausungen gebildet, nachgebildet den Fächern in Turmbau zu Babel von Pieter Bruegel, dem Älteren. Ubus vogelartiger Kopf ist geneigt, ein schwarzes Loch figuriert als Auge, die herausragende Nase ist ein weißer Knochen, die Topfform des Kopfes verstärkt den Eindruck, der Imperator ähnle einem Schneemann. Bedeckt, nur wenig bedeckt, ist der Imperator durch einen grün-blauen Umhang, zwischen Haar und Kleidungsstück changierend. Kein Hermelinfell! Der Schattenwurf, den Max Ernst in seiner Frühphase in Anlehnung an Chiricos metaphysische Malerei gern einsetzt, ist auf diesem Bild vage geworden.

Die Steigerung ins Stürzende macht die Position bedenklich, riskant, sogar fatal. Ubu hebt Arme und Hände hoch - er ist lächerlich und hilflos zugleich. In der Geste scheint Ubu nach Gleichgewicht zu suchen und zu fragen: was soll ich denn bloß machen? Der Kopf wackelt. Der Turm droht einzustürzen. Der Imperator tanzt bzw. schwankt auf Sand. Die Tage seiner Herrschaft sind gezählt. Der Kaiser Ubu ist noch lächerlicher als der König Ubu. Bewegt sich Jarrys gewalttätiger und grotesker König Ubu in einem imaginären Polen, so bildet der lächerliche Imperator ein Anschauungsobjekt, das die deutschen und französischen Avantgarden gemeinsam verspotten können, nämlich den bürgerlichen Fortschrittsglauben, den Glauben an die Triumphe der Zivilisation. Dies ist ein Drittes, das sie ablehnen. Zugleich bildet die Figur, nicht zuletzt durch den Verweis auf die Babelsche Sprachverwirrung, ein Bild der Konflikte zwischen Kulturen und der Konflikthaftigkeit von Kultur selbst. Bereits Jarry benutzt seinen Père Ubu und seinen $U b u$ roi nicht einfach, um sich über lächerliche Pauker oder Herrscher, also über die anderen lustig zu machen, sondern verkörpert immer auch selbst den grotesk-gewaltsamen Ubu.

Dieses Dritte ist nicht nur die lächerlich gemachte bürgerliche Kultur der anderen. Die Monsterengel der dreißiger Jahre mit den erhobenen Armen und Händen wissen weder ein noch aus. Sie wissen nicht, wohin mit ihrer Aggressivität und Wut. Sie sind hilflos und leiden auch. Es handelt sich um 
eine Patheinformel, insofern als die erhobenen Hände und der leere Blick immer auch einen eigenen Zustand des Konflikts, des Selbst und der eigenen Kultur, abbilden.

L'Ange du foyer (1937) ist im Kontext des Spanischen Bürgerkriegs entstanden. Der Titel spielt auf die Komödie L'Ange du foyer an, ein Film von Léon Mathot von 1936, den Mathot nach der gleichnamigen Komödie von Robert de Flers (1905) gedreht hat: Ein Freund, der Hausengel, rettet die Ehe zwischen einem donjuanesken Ehemann und dessen Ehefrau. Eine heilende komische Dreierkonstellation. Max Ernst hat sich mit Ubu Imperator wie auch L'Ange $d u$ foyer in eine komödiantische Traditionslinie Frankreichs gestellt. In beiden Fällen kippt das Komische ins Furchtbare. Der „Hausengel“ ist ein tobendes Trampeltier. Es hat mit ihm verwachsen eine kleinere Mitkreatur, mit der es kämpft. Kein Drittes schlichtet oder versöhnt. Die Gewalt ist nicht das, was außerhalb des vernünftigen Menschen lokalisiert ist und von den Menschen guten Willens mit den Mitteln der Vernunft bekämpft werden kann. Sie ist vielmehr in uns. Die Traumatisierung, die zu mühsam unterdrückter Wut gegen sich selbst und potentiell gegen andere führt, ist das, was Deutsche und Franzosen verbindet.

Anders als der klassizistische Stil, dessen Mitriss eine Friedensordnung konfiguriert, zu der Deutsche und Franzosen gehören, verkörpert die durchgehende Geste der aggressiven Hilflosigkeit eine Versehrtheit und ein Geschlagensein von Deutschen und Franzosen in Zeiten des Krieges und der Gewalt. Die Bilder gehorchen nicht dem Indizienparadigma, wonach Deutsche und Franzosen identifiziert und geschieden werden könnten. Vielmehr verwirklicht die Reihe der Bilder Kontextualisierungen, die durch eine Patheingeste verbunden sind und die Verdüsterung der Welt materialisieren. Die Bilder bieten Widerstand als verstörende halb organische, halb anorganische Wesen. Sie sind affektiv unscharf.

\section{Der und das Dritte und das Fehlende}

Der Akzent ist immer auch ein Mal des Verfehlens und des Fehlens - der Sprecher verfehlt die Perfektion, es fehlt die perfekte Aussprache. Es ist die Versehrtheit und das Fehlen, die Körperteile zu expressiven Masken des Transkulturellen machen. Körperteile können eine sakrale Qualität in Vorgängen der Friedensstiftung gewinnen. Es handelt sich um eine magische Aneignung der Eigenschaften eines anderen. Dies gilt auch im Fall der Misshandlung im und nach dem Krieg. Die während der Befreiung geschorenen Französinnen werden ihrer Haarpracht beraubt. Die geschorenen Haare sollen ein Bild der Schande abgeben, aber das Bild der Schande wird ein Schandbild für die Misshandler.

Möglich macht dies der Eingriff eines Dritten. Das berühmte Foto „La femme tondue“ stammt von einem Dritten, von Robert Capa, geboren als Endre Friedman in Budapest. Es zeigt ungeschminkt die Gewalt, die Frauen angetan wird, die der sogenannten „horizontalen Kollaboration“ beschuldigt werden. Der deutsche Besatzer ist nicht mehr greifbar. An seiner Stelle hält die Meute, 
die selbsternannten Vertreter der Volksjustiz, sich an der wehrlosen Frau schadlos. Abwesend ist auch das Haar, seine Schur gilt dem deutschen Besatzer; die Frucht der verbotenen Liebe, das Baby, ist hingegen anwesend.

Im Chanson „La tondue“ von Brassens ist der kahle Schädel mit der verfemten Sprache verknüpft. Der Vortragende ist der nicht eingreifende Zuschauer der Szene, der sich schämt.

\footnotetext{
La belle qui couchait avec le roi de Prusse [...],

à qui l'on a tondu le crâne rasibus [...],

son penchant prononcé pour les „Ich liebe dich“ [...],

Lui valut de porter quelques cheveux postich's [...].

Les braves sans culott's et les bonnets phrygiens [...],

Ont livré sa crinière à un tondeur de chiens [...].
}

Brassens, der 1943/44 Zwangsarbeiter in Deutschland war, hält nicht nur die in Frankreich eher positive Erinnerung an Friedrich II. aufrecht, er führt auch einen Satz auf Deutsch ein. Tonaufnahmen bezeugen, wie er sich für seine fehlerhafte Aussprache von „Ich liebe dich“ entschuldigt. Er spricht Deutsch mit sehr starkem Akzent.

Ist der Zuschauer nicht mutig, steht er der Armen nicht bei, so heftet er sich doch einen abgetrennten Haarteil ans Revers, nicht als Trophäe des Siegers, sondern als Liebeszeichen, als Zeichen der Erinnerung.

\footnotetext{
Les coupeurs de cheveux en quatre m'ont fait peur [...].

Quand, pire qu'une brosse, elle eut été tondue [...],

J'ai dit: „C'est malheureux, ces accroch'-cœur perdus“ [...].

Et ramassant l'un deux qui traînait dans l'ornière [...],

Je l'ai, comme une fleur, mis à ma boutonnière [...].
}

Das abweichende Mal, das eine stumme expressive Maske ist, besteht aus einem abwesenden Körperteil, den Haaren. Aus der Trophäe, die die selbsternannten Vertreter der Volksjustiz sich angeeignet haben, wird ein Talisman. So gelingt es, der Erniedrigten die Würde zurückzuerstatten.

Das Ertönen des tabuisierten Deutsch und die Körpergeste des Aufhebens und Ansteckens der Locke sind Ausdrucksformen, die die riskante Fürsprache eines Franzosen verkörperlichen. Solche Vorgänge setzen voraus, dass körperliche Partialobjekte Dritte sind, die stellvertreten und Eigenschaften des Fremden magisch verkörpern. Die Körperteile bewahren Wissen von der Verbindung mit einem Fremden, hier von dem deutschen Besatzersoldaten, auf. Das Partialobjekt ist berührbar, ein nicht nur symbolischer Körperteil. Entscheidend ist nun das, was das Kunstwerk mit dem Fetisch macht: Die Schur, die die Frau erniedrigt, demütigt, zum Vieh herabwürdigt, kippt im Blick des Betrachters des Fotos oder des Zuhörers des Chansons. Das Chanson verwandelt die Besatzersprache in Liebessprache, und die Trophäe, die Beute zum Talisman. In verkörpernden Kunstformen wie dem Chanson tönt es nicht nur durch, es fließt hindurch und belebt das Objekt. 


\section{Der Transleib als Übergang: Koexistenz mit Scheppern}

Wenn ein künstlerisches Medium die Störung nicht aufnehmen, nicht verkörpern kann, ist ein wesentliches Element des Kulturkontakts nicht fühlbar. Das Bild kann den Akzent nicht übermitteln. Der Leser hört die Figuren von Siegfried nicht sprechen, sei es mit oder ohne Akzent. Dies ändert sich mit dem Medium Tonfilm. Im Film sind sowohl die Aussprache hörbar wie das Aussehen sichtbar. Auch die Abweichungen. Der transkulturelle Sprachwechsel ist eine gedankliche wie sinnliche Herausforderung. Er kann auch im Film verleugnet werden, indem die stimmige Verbindung zum Leib aufgelöst wird. Die Synchronisation räumt dem Hören der eigenen Sprache Vorrang ein, sie setzt die lautliche Übersetzung ein, um das Fremde zu vertreiben. Der Preis ist die körperliche Inkongruenz von Stimme und Gesicht. Das schmerzhafte und gleichwohl unthematisierte Auseinanderklaffen von Hören und Sehen in der furchtbaren Praxis der Synchronisation von Filmen ist die Regel auf den Kanälen des privaten wie öffentlich-rechtlichen Rundfunks in Deutschland und Frankreich - im Unterschied zur Praxis der Untertitelung in den Niederlanden und den skandinavischen Ländern. Die Synchronisation beraubt die Schauspieler ihrer Stimme. Die sie ersetzende Stimme stimmt nicht mehr mit der Mimik überein. Das Übersetzen ist zwar unvermeidbar, notwendig. Bliebe es aber auf das Schriftliche, also auf Untertitel, beschränkt, könnten Gesichtsausdruck und Sprache weiterhin korrespondieren - und die Zuschauer sich mit Gewinn in Fremdsprachen einhören. Das Grimassieren in synchronisierten Filmen wird indes gar nicht mehr bewusst wahrgenommen. Auch wenn Schauspieler selbst ihre Synchronstimme sprechen (zum Beispiel Romy Schneider, Vicky Krieps), ist die Nicht-Deckung von Stimme und Mimik nicht aufgehoben.

Konträr zur Synchronisation steht die Entscheidung, Schauspieler in ihrer Muttersprache und in einer Fremdsprache sprechen zu lassen. Sie sprechen dann sowohl ohne wie auch mit Akzent. Dies ist der Fall in Filmen wie $L a$ Grande Illusion (1937) von Jean Renoir, Frantz (2016) von François Ozon und auch Transit (2018) von Christian Petzold. In diesen Filmen reden Franzosen im deutschen Kontext Deutsch mit Akzent, Deutsche im französischen Kontext Französisch mit Akzent. In La Grande Illusion spricht nicht nur Erich von Stroheim (von Raufenstein) mehrere Sprachen, auch Jean Gabin (Maréchal) spricht Französisch und Deutsch. Die Anlage wird chiastisch, jeder redet sowohl in seiner Muttersprache wie - mit Akzent - in seiner Zweitsprache. Über eine gewöhnliche Stellvertretung - als Diplomat, als Händler - hinausgehend stellen die Akteure unvertretbar ihren Leib zur Verfügung, der das defiziente Sprechen auferlegt.

In einigen Filmen verbergen sich Fortschreibungen der zentralen deutschfranzösischen Texte ${ }^{23}$, so in Frantz, aber auch in Allemagne, année 90 neuf zéro

23. Mehr dazu vom Verfasser: Verkörperungen des Dritten im Deutsch-Französischen Verhältnis. Die Stelle der Übertragung, Paderborn, Fink, 2018. 
von Jean-Luc Godard (1991). In Allemagne, année 90 neuf zéro haben Delphine de Staël und Zelten, eine Figur aus dem Siegfried, einen Auftritt. Sie übersetzen gemeinsam. Nicht Identifikation und Ersetzbarkeit des einen durch den anderen sind Programm, sondern die Arbeit des Übersetzens.

Eine andere Möglichkeit wählt François Ozon in Frantz. Der überlebende Täter Adrien soll dessen Stelle bei den Eltern und der Braut von Frantz einnehmen. Dass der französische Soldat den von ihm im Schützengraben getöteten Deutschen nicht bei der Braut und deren Schwiegereltern ersetzen kann, macht den Unterschied vom Film Frantz zu seiner Vorlage L'Homme que j'ai tué (1925) von Maurice Rostand und der akzentfreien Verfilmung von Lubitsch unter dem Titel Broken Lullaby (1932) aus.

Die Violine bzw. die auf ihr gespielte Musik bilden in L'Homme que j'ai tué und Frantz eine sächliche Kontinuität zwischen beiden. Das Instrument wandert zwischen den Männern. In Frantz spielt Adrien auf Frantz' Violine, er verliert die Fassung, er bricht zusammen. Die Laute ohne Sprache lösen eine eigene freilich missverständliche Resonanz aus. Sie restituieren nicht die Wahrheit. Das Sprechen ist nicht in derselben Weise mit Kontrollverlust verbunden. Der Akzent ist eine expressive Maske, eine Ausdrucksform. Der Akzent ist nicht einfach abstreifbar, aber er ist in anderer Weise unwillkürlich als Lachen und Weinen. Er besteht auf der Fühlbarkeit der Erstsprache in der Zweitsprache.

In Frantz tritt die Metonymie, die Benachbarung und das Knirschen des Akzents, wieder in ihr Recht. Paula reist Adrien nach, sie spricht jetzt Französisch, mit Akzent. Die Verbindung mit Adrien kommt nicht zustande. Adrien ersetzt den von ihm getöteten Frantz nicht. Paula erfındet eine barmherzige Lüge für ihre Schwiegereltern. Der semantische Gehalt, die Botschaft der Briefe, täuscht, genauso wie die Botschaft der Bilder. Der Film lügt, so ist etwa der Rückblick erfunden, als die Freunde, noch vor dem Krieg, den Louvre besuchen. Die beiden sind sich, abgesehen von der fatalen Begegnung im Schützengraben, nie begegnet. Das Indizienparadigma führt in die Irre, sprachlich und bildlich.

Die Zweisprachigkeit ohne und mit Akzent steht für eine Unebenheit, die wenn nicht wahr, so doch integer ist. Sie steht für Versehrtheit und Unersetzbarkeit. Die Zweisprachigkeit zieht allerdings Untertitel nach sich.

Auch Transit von Anna Seghers spielt mit dem Phantasma der Ersetzbarkeit und mit Versehrtheit. Franz will Georg Weidel bei dessen Frau ersetzen. Der Film Transit von Christian Petzold lebt von der Zweisprachigkeit mit Akzent. In einer chiastischen Konstellation spricht der Exilierte Georg Französisch mit Akzent, der junge Driss Deutsch mit Akzent. Die Romanhandlung ist aus dem Jahre 1940 in das heutige Paris und Marseille übertragen, die Stimme ist diejenige des Zustands des Transit, gestern, heute, morgen. Die Frau mit den Hunden, ebenfalls eine Exilierte, stellt auf Deutsch das MUCEM und Riciotti vor, also Gegenwärtiges, bevor sie sich genau an diesem Schauplatz in die Tiefe stürzt.

Der Film Transit stellt die bildnerischen Elemente aus Seghers' Roman in einen neuen zeitlichen Rahmen. Zudem macht er Fremdheit lautlich spürbar. Petzold hat die Abweichung der Aussprache, die Versehrtheit noch gesteigert, 
da er in Nahaufnahme auch das Gesicht von Franz Rogowski zeigt, mit der vernähten Gaumenspalte. Er spricht sehr gut Französisch, aber beide Sprachen mit einem sehr leichten Sprachfehler.

Der Akzent ist in La Grande Illusion oder in Frantz oder in Transit nicht mehr Anzeichen der Nicht-Zugehörigkeit einzelner Menschen, sondern die expressive Maske der conditio humana: fast jeder Mensch, der eine Zweitsprache spricht, hat einen Akzent. Der Akzent verkörpert und bezeugt die Öffnung zum Anderen. Er ist dasjenige, was verbindet, nicht als semantischer Gehalt, sondern als bedeutungsloser Laut. Dies kann er dann mit den tendenziell inhaltsleeren Stimmen in verkörpernden Kunstformen - Lied, Oper, Film, Installation, Performance - teilen. Sofern solche Kunstformen dem fremden Laut einen Platz geben, machen sie die Stimme zum Medium der Berührung.

Verkörpernde Kunstformen, die verschiedene Sinne und Kunstformen zusammenführen, wie Lied, Theater, Performance und eben auch der Film, haben den Vorzug, Transkulturalität stimmlich und bildlich wahrnehmbar zu machen.

\section{Schluss}

Ich schließe auf Französisch: l'accent, le signal le plus sensible du masque expressif, marque, tire une frontière; il transgresse cette frontière en reliant deux mondes.

Die nur auf den ersten Blick paradoxe Schlussfolgerung ergibt sich wie von selbst: Trennende Grenzkörper entstehen immer dann, wenn das Störende ausgeschlossen, ausgeblendet wird. Verbindende Grenzkörper, Schwellen bilden sich immer dann, wenn Mangelhaftes und Irritierendes auftreten darf, eben das, was überträgt und dem Übertragenen vorausgeht.

Die Wahrnehmung dessen, was überträgt, ermöglicht es, den lautlichen, stilistischen und bildlichen Körper, der auch lebendiger Leib ist, zu erfassen, der Deutsche und Franzosen verbindet.

Die Literatur zeigt dabei ein strukturelles Defizit auf. Ihr fällt es schwer, der expressiven Maske, etwa dem Akzent, Präsenz zu verschaffen.

Verkörpernde Kunstformen sind besser geeignet, Versehrtheit zu übertragen. Ob Brassens in seinem Eintreten für die geschorenen Frauen, ob Ozon in seinem Verständnis für die Eltern, die Braut und den unschuldig-schuldigen Täter, sie schaffen Werke des Mitgefühls. Dieses Mitgefühl braucht den Akzent, die Defizienz und die Störung, ein fehlerhaftes Hinübergleiten. Kunstformen wie der Film haben neue Möglichkeiten ergriffen, der abweichenden expressiven Maske einen Platz zu geben.

Ich empfinde Dankbarkeit für die Zuneigung für die Geschlagenen, die in ihnen zum Ausdruck kommt. 
